Contributions to Game Theory and Management, XIII, 121-131

\title{
Games With Fuzzy Payment Matrix
}

\section{Vladimir G. Chernov}

\author{
Vladimir State University named after Alexander Grigorievich and Nikolai Grigorievich \\ Stoletovs ( VlSU), \\ Department of Computer Engeneering and Control Systems, \\ Gorky str. 87, Vladimir, 600000, Russia \\ E-mail: vladimir.chernov44@mail.ru
}

\begin{abstract}
We consider a method for solving an antagonistic game with a fuzzy payment matrix based on converting fuzzy estimates of the consequences of possible strategies into an integral estimate in the form of an equivalent fuzzy set with a triangular membership function. The method does not impose restrictions on the type of membership functions for fuzzy elements of the payment.
\end{abstract}

Keywords: fuzzy set, fuzzy number, membership function, fuzzy payment matrix

\section{Introduction}

Antagonistic game as a model of a conflict situation can be set by a triple

$$
\left\langle A=\left(a_{i}: i=\overline{1, I}\right), B=\left(b_{j}: j=\overline{1, J}\right), R(A B)\right\rangle,
$$

where for $A, B$ - set of players' strategies, $R$ - payment matrix. The process of constructing a payment matrix is one of the most important and complex stages of game-theoretical modeling of the decision-making situation. In the process of building a payment matrix there are a number of problems (Seagal, 2011):

1. These are the problems associated with the assessment of the representativeness of sample data sets, on the basis of which the values of the elements of the payment matrix are determined;

2. Evaluation of the truth of the values obtained as a result of statistical observations;

3. The statistics reflect the past state of the decision-making situation, hence the question of their relevance to the present. Suffice it to recall the nonreproducibility of economic conditions in economic systems;

4. Expert assessments are fundamentally characterized by uncertainty, which is not reflected in the traditional procedures for the construction of payment matrices;

5. Sets of players' strategies have a complex structure and it is almost impossible to prove the completeness of these sets.

Classical game theory is based on the assumption that players have complete information about the set of possible strategies and the payment matrix, the elements of which are point numbers, which is essentially a simplified model of the real situation. Obviously, because of the above difficulties, it is very difficult to rely on an accurate knowledge of the elements of the payment matrix, and most likely they represent approximate estimates of the decision-making situation. In this regard, 
the situations when the elements of the payment matrix $R(A, B)$ are fuzzy numbers are considered more and more often, for example, (Bector and Chandra, 2010; Falomkina, 2009; Higast and Klir, 1983; Orlovsky, 1976; Zaichenko, 2010) and others. In several studies (Chang, 1994; Sahoo, 2017; Stalin and Thirucheran, 2015; Qui et al., 2018), in order to find the best solution, the fuzzy elements of the payment matrix are replaced by their modal values, thus making the fuzzy game clear. Proposed in (Dutta and Gupta, 2006; Seikh et al., 2015; Vasilevich, 2010; Vovk, 2012; Seraya and Katkova, 2012) methods are intended only for solving games in which fuzzy elements of payment matrices have piecewise linear membership functions. In the absence of a saddle point in research (Bector and Chandra, 2010; Campos, 1989; Cevikel and Ahlatcloglu, 2010) they adhere to the classical scheme, solving the game in mixed strategies, while the game with a fuzzy payment matrix is reduced to a clear game, or they use methods of fuzzy linear programming, because of the complexity of which only triangular membership functions are considered. It should be noted that the use of mixed strategies involves multiple implementation of the game with unchanged values of the initial parameters. If the game parameters are unclear, it means that the game parameters can be changed, which contradicts the conditions for using mixed strategies. In this paper, we propose a solution to a fuzzy antagonistic game no restrictions on the type of membership functions for fuzzy elements of the payment matrix and without a transition to a clear statement.

\section{Game Formulation}

As noted above, the formulation of an antagonistic game begins with the construction of sets of player strategies and a payment matrix. When building a fuzzy payment matrix (FPM), it is necessary to determine how fuzzy numbers (FN) will be set. First of all, it is necessary to choose the type of membership function (MSF) of a fuzzy number, because by choosing one or another type of MSF, we formulate our idea of the degree of uncertainty of the decision-making situation. For example, in a fuzzy spreadsheet FuzzyCalc (Chernov et al., 1998) the MSF library has the following options, which model different levels of uncertainty.To prove, we calculate the powers of fuzzy sets with reduced MSF by the formula proposed by De Luca and Termini (Dubois and Prade, 1980) (Fig. 1, Table 1)

$$
|W|=\sum_{x \in X} \mu(x),
$$

where $|W|$ - the power of fuzzy set and $\mu(x)$ - the membership function of a fuzzy set. Power in this case is treated as an indicator of fuzziness.

Table 1. Membership options

\begin{tabular}{|c|c|}
\hline Type of MSF & W \\
\hline Peak & 8.9 \\
\hline Triangle & 12.8 \\
\hline Tent & 14.1 \\
\hline Trapeze & 18 \\
\hline
\end{tabular}

In the general case, there are ample opportunities to represent the uncertainty of the values of the FPM elements. However, the constraining factor here will be 


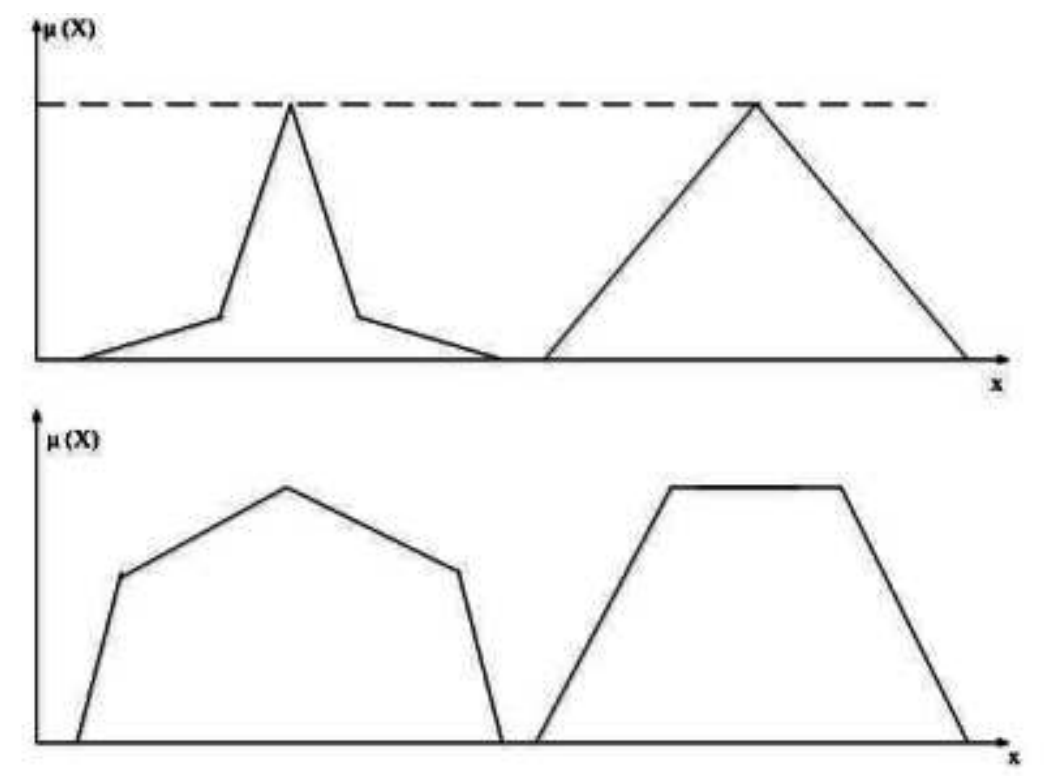

Fig. 1. The variants of MSF: the peak, triangle, tent, trapeze

the difficulties that arise when performing the necessary transformations over the fuzzy elements of the FPM.

Two options are possible:

1. All elements (fuzzy numbers) of FPM have the same MSF;

2. When determining the elements of the FPM can be used different MSF.

Choosing the same membership functions, for example, those that correspond to the $L R$-representation of fuzzy numbers, we simplify the execution of arithmetic operations that may be required later. This option, in addition to this, allows to automate the construction of the payment matrix, since it is enough to choose a specific type from the library of standard MSF, specify a modal value and a deviation, and further procedures can be performed without the participation of the user, of course, with the appropriate software.The second option is more difficult to implement, requires more complex options for performing arithmetic operations (calculations using $\alpha$-level sets or the Zadeh generalization principle).

The classical scheme of the solution (finding the best strategies of the players) of the antagonistic game is carried out in several stages:

1. Check of possible strategies for domination;

2. Check for saddle point;

3. Finding the best strategy.

It should be noted that at all stages need a fuzzy comparison operation, which has significant features. The most simple comparison of FN is performed if their MSF do not intersect and is much more difficult when intersecting (Chang, 1994, Rao and Shankar, 2012). 
The comparison operation can be viewed as establishing a linear order relation between the elements of a certain set, in our case it is a set of fuzzy numbers. The proposed method (Chernov, 2018) for comparing fuzzy numbers consists in proving the existence of a fuzzy hypothesis about the possibility of constructing a linear order relation of a given type of "more" or "less" for some set of fuzzy numbers.

Definition 1. The fuzzy hypothesis is formalized by two fuzzy sets defined on the set of possible values of fuzzy numbers that make up the FPM and represent estimates of the possibility of assigning the FPM elements to the set of minimum or maximum values (Chernov, 2018) (Fig. 2).

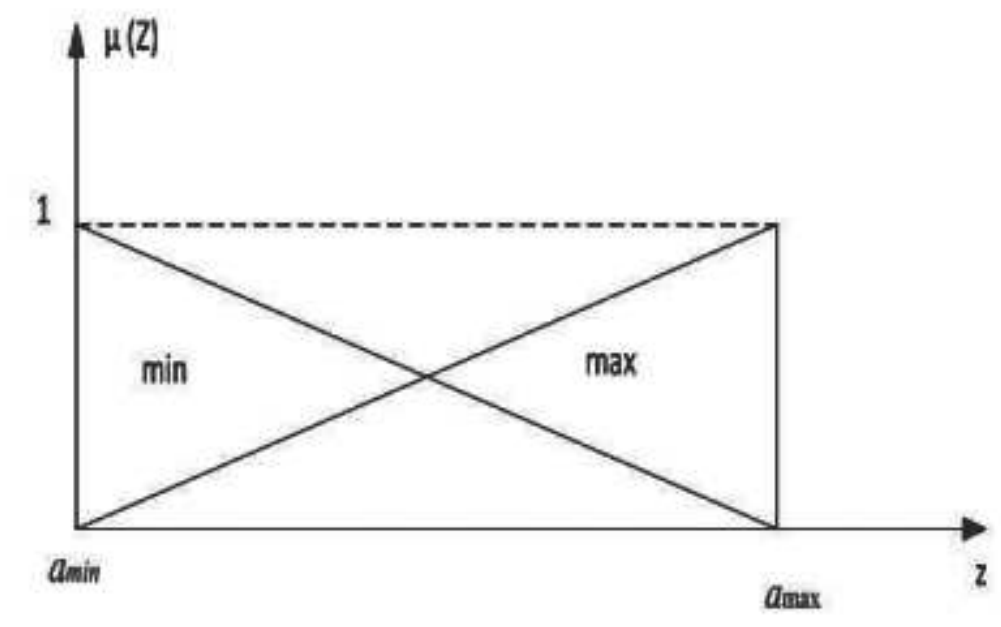

Fig. 2. The FPM elements to the set of minimum or maximum values

$$
\begin{gathered}
\widetilde{M}=\left\{\mu_{\min }(z), \mu_{\max }(z), z \in\left[a_{\min }, a_{\max }\right]\right\} \\
\widetilde{M}=\left\{\begin{array}{c}
\mu_{\max }(z)=\left(a_{\max }-z\right) /\left(a_{\max }-a_{\min }\right), \\
\mu_{\min }(z)=1-\mu_{\max }(z), z \in\left[a_{\min }, a_{\max }\right]
\end{array}\right\}
\end{gathered}
$$

$\left[a_{\min }, a_{\max }\right]-$ area of definition of elements of the FPM.

Theorem 1. The problem of establishing a linear order relation on the set $M$ is proposed to be solved by constructing a map of the set of fuzzy numbers on the set $M$, using the intersection operation

$$
\widetilde{S}=\mu_{\widetilde{a}_{i j}}(x) \cap \mu_{\widetilde{M}}(x), x \in\left[a_{\min }, a_{\max }\right] .
$$

Chernov, 2018)

Lemma 1. The intersection operation is most often formalized as a min operation.

$$
\widetilde{S}=\min \left(\mu_{\widetilde{a}_{i j}}(x), \mu_{\widetilde{M}}(x)\right), x \in\left[a_{\min }, a_{\max }\right] .
$$


Lemma 2. Alternative formalizations of the intersection operation is the product (Prod) of the corresponding membership function

$$
\widetilde{S}=\mu_{\widetilde{a}_{i j}}(x) \cap \mu_{\widetilde{M}}(x)=\mu_{\widetilde{a}_{i j}}(x) * \mu_{\widetilde{M}}(x), x \in\left[a_{\min }, a_{\max }\right] .
$$

and the so-called boundary intersection function

$$
\widetilde{S}=\mu_{\widetilde{a}_{i j}}(x) \cap \mu_{\widetilde{M}}(x)=\max \left[\mu_{\widetilde{a}_{i j}}(x) \cap \mu_{\widetilde{M}}(x)-1.0\right], x \in\left[a_{\min }, a_{\max }\right] .
$$

It seems, that the interpretation of the intersection operation as a product or boundary intersection is more consistent with the content of the problem of comparing fuzzy numbers.

Resulting two fuzzy sets $\widetilde{S}\left(\widetilde{a}_{i j}\right)$ and $\widetilde{S}\left(\widetilde{a}_{i k}\right)$.

In addition, for fuzzy elements need to determine the criterion for assessing the truth of the constructed relationship.

The membership functions of these sets can be interpreted as the distribution of the truth of the fuzzy hypothesis that in a pair $\left(\widetilde{a}_{i j}, \widetilde{a}_{i k}\right)$ one of the elements will be, for example, minimal.

Definition 2. The values $\alpha_{i j}=\max \left[\mu_{\widetilde{S}\left(\widetilde{a}_{i j}\right)}(x)\right]$ and $\alpha_{i k}=\max \left[\mu_{\widetilde{S}\left(\widetilde{a}_{i k}\right)}(x)\right]$ can be considered as an estimate of the truth of the corresponding hypothesis.

By following the appropriate comparison procedure described, we can determine the presence of a saddle point, by identifying not useful strategies to remove them from consideration.

The final step is to find the best strategies of the players. In the traditional formulation, when the payment matrix consists of point numbers, it is proposed to use mixed strategies.

There are criticisms of mixed strategies.The first parties a zero-sum game it is rational actors and their choice of strategies through the mechanism of random selection is hardly possible in practice, unless, as is noted by E. S. Ventzel (Ventzel, 2004) this is not the way to lead the enemy into confusion, it is noted also that the mechanism of random selection strategies to the substance of the tasksis not relevant. In its original version, the model of the game does not include the element of chance, but its introduction and the theoretical - probable approach to the definition of the criterion of the quality of the solution as a mathematical expectation of winning, makes sense only when the individual acts of the game are repeated many times and independently. In the case of a single act of the game, the probability criterion loses its meaning. It should also be noted that the multiple implementation of a single act of the game involves the immutability of the values of the elements of the payment matrix.

If a game with a fuzzy payment matrix is considered, it assumes that the values of its elements can vary within the respective carriers, i.e. for each implementation of the game the conditions can change, which obviously contradicts the initial prerequisites for the definition of mixed strategies. In the known variants of the solution of the considered problems with fuzzy initial data, either particular forms of uncertainty representation are considered, or in some way the fuzzy problem is reduced to a clearstatement.

In the conditions of uncertainty of the task of elements of the payment matrix, there are enough reasons to believe that the player does not know reliably what 
strategy the enemy will choose. Although by definition the enemy must act rationally, it is acceptable given the uncertainty of his choice, to consider it as "nature". Then, if we consider the strategic game as some analogue of the game with nature, we can recall the principle of Bayes, according to which, with a known distribution of probabilities of the states of nature, the player will have at least one pure strategy that allows you to get the best result. In the case of FPMs, the vagueness of its elements is a way of formalizing uncertainty.

An analogue of the Bayes principle in relation to the game with a fuzzy payment matrix can be formulated as follows.

Theorem 2. In a game with a given type of fuzzy values of the elements of the payment matrix, players will have at least one pure strategy that provides the best result.

Choose an arbitrary strategy of the first player of the $a_{j}$, while the second player can apply any of the strategies $b_{k}$,

$$
\begin{array}{|c|c|c|c|c|c|}
\hline & b_{1} & b_{2} & \cdots & \cdots & b_{m} \\
\hline a_{k} & \widetilde{m}_{k 1} & \widetilde{m}_{k 2} & \cdots & \cdots & \widetilde{m}_{k m} \\
\hline
\end{array}
$$

Theorem 3. The statement that the first player does not know exactly the choice of the second is equivalent to the statement that the second player will apply "strategy $b_{1}$ or $b_{2}$ or... ... or $b_{m}$ " which can be formalized as a Union

$$
\bigcup_{j=1}^{m} \widetilde{m}_{k j}=\widetilde{R}_{k}=\left\{\bigcup_{j=1}^{m} \mu_{k j}(x)\right\},
$$

because the choice of the second player is not known to the first.

Proof (of Theorem 3). These proposals can be justified by analogy from the theory of probability. If $A$ and $B$ are two arbitrary events that can intersect and, then the ratio is true $P(A+B)=P(A)+P(B)-P(A B)$. If $A$ and $B$ are independent, then $P(A B)=P(A) P(B)$. Respectively,

$$
P(A+B)=P(A)+P(B)-P(A) P(B) .
$$

In one interpretation of the membership functions it is considered as a distribution of the possibilities of occurrence of some events. Then, if in the ratio (2) probability to replace the membership function, we obtain one of the alternative forms of Union

$$
\mu_{A \cup B}(x)=\mu_{A}(x)+\mu_{B}(x)-\mu_{A}(x) \mu_{B}(x),
$$

and from the ratio (2) - an alternative form of intersection, the so-called Prod (Piegat, 2013)

$$
\mu_{A \cap B}(x)=\mu_{A}(x) \mu_{B}(x) .
$$


Definition 3. As a result, we get a fuzzy set (number) that determines the possible results of the first player when he chooses the strategy $a_{k}$ and some choice of the second. If we perform transformations (1) for all strategies of the first player, we get a set of fuzzy sets (numbers)

$$
\widetilde{R}(A)=\left\{\widetilde{R}_{k}: k=\overline{1, n}\right\}=\left\{\cup_{j=1}^{m} \mu_{j k}(x): k=\overline{1, n}\right\} .
$$

In general, fuzzy sets $\widetilde{R}_{k}$ and, accordingly, $\widetilde{R}(A)$ have membership functions of any kind and comparison of the corresponding fuzzy numbers in order to identify the best strategy will be quite a difficult task. Therefore, it is advisable to give the form of fuzzy sets (membership functions) to a single variant.As such a transformation, we can propose the operation FztoTriangle.

Definition 4. FztoTriangle replaces an arbitrary fuzzy set $\widetilde{R}_{k} \rightarrow \widetilde{R}_{k}^{T r}$ with a fuzzy set with an equivalent triangular membership function, in which the left and right boundaries, as well as the center of gravity coincide with similar indicators of the original membership function, and the maximum value of the membership function should be preserved.

The FztoTrianle transformation is based on fairly simply relationship. The initial data for constructing an equivalen fuzzy set with a triangular membership function are: the boundaries of the carrier and the coordinate of the center of gravity of the fuzzy set obtained as a result of transformation FztoTriangle, which we denote as $\left[z_{\min }, z_{\max }\right], z_{C G}$ - coordinate of the center of gravity. Since in this case the maximum value of the membership function of the equivalent fuzzy set should be 1 , then the triangular membership function is uniquely determined by the triple $\left[z_{L}=z_{\min }, z^{*}, z_{R}=z_{\max }\right]$, where $z^{*}$ is the unknown coordinate of the maximum of the membership function.

Definition 5. The value of $z^{*}$ can be determined on the basis of the known relation for determining the coordinates of the center of gravity of a triangle with the coordinates of vertices $\left(z_{L}, z^{*}, z_{R}\right)$

$$
z_{C G}=\frac{1}{3}\left(z_{L}+z^{*}+z_{R}\right)
$$

When calculating according to relation (3) for some values of $z_{L}, z_{C G}, z_{R}$ the value $z^{*}>z_{R}$ can be obtained, which is impossible accordingto the conditions for determining the membership function. Therefore, when calculating the $z^{*}$ value, it is necessary to introduce the corresponding restriction. Then

$$
z^{*}=\left\{\begin{array}{c}
3 z_{C G}-z_{L}-z_{R}, z^{*}<z_{R} \\
z^{*}=z_{R}, z^{*} \geq z_{R}
\end{array}\right\}
$$

Another situation is also possible, when calculating by the ratio (3) for some combination of values $z_{L}, z_{C G}, z_{R}$, it will be obtained that $z^{*}<z_{L}$, which is also impossible under the conditions of constructing membership functions. In this case the following restriction must be applied

$$
z^{*}=\left\{\begin{array}{c}
3 z_{C G}-z_{L}-z_{R}, z^{*}>z_{L} \\
z^{*}=z_{L}, z^{*} \leq z_{R}
\end{array}\right\}
$$


We can show that this transformation does not change the logic of the game. To compare fuzzy numbers, point estimates can be used (Yager, 1977), the values of which depend on the position of the number on the numerical axis. The more to the right a fuzzy number is located, the greater its point estimate. The FztoTriangle transformation preserves the relative position of fuzzy numbers representing an estimate of the result of choosing a particular strategy. Accordingly, for equivalent fuzzy numbers obtained after the FztoTriangle transformation, the ratio between the point estimates will remain unchanged.

Definition 6. The possible result of the first player using some strategy $a_{k}$, if the choice of the second is not known, can be represented by the equivalent fuzzy set $\widetilde{R}_{k}^{T r}$. Similarly, any strategy of the second player $b_{l}$ can be matched by a fuzzy number $\widetilde{H}_{l}^{T r}, l=\overline{1, m}$.

$$
\left|\begin{array}{c}
a_{1} \\
a_{2} \\
\vdots \\
a_{n}
\end{array}\right| \rightarrow \widetilde{S}_{1} \rightarrow\left|\begin{array}{c}
\widetilde{R}_{1}^{T r} \\
\widetilde{R}_{2}^{T r} \\
\vdots \\
\widetilde{R}_{n}^{T r}
\end{array}\right|,\left|\begin{array}{c}
b_{1} \\
b_{2} \\
\vdots \\
b_{n}
\end{array}\right| \rightarrow \widetilde{S}_{2} \rightarrow\left|\begin{array}{c}
\widetilde{H}_{1}^{T r} \\
\widetilde{H}_{2}^{T r} \\
\vdots \\
\widetilde{H}_{m}^{T r}
\end{array}\right| .
$$

Definition 7. The first player as the best will choose the strategy $a_{k} \rightarrow \max _{k} \widetilde{R}_{k}^{T r}$, $k=\overline{1, n}$, and the second $-b_{l} \rightarrow \min _{l} \widetilde{H}_{l}^{T r}, l=\overline{1, m}$.

The best strategy can be determined either using point estimates or a method based on the fuzzy preference hypothesis. Mark, that both methods give unambiguousand coinciding results, but the point estimation method is more cumbersome in computational terms.

Definition 8. The equilibrium result is defined as the intersection

$$
\widetilde{\gamma}=\min \widetilde{H}_{l}^{T r} \cap \max \widetilde{R}_{k}^{T r} .
$$

These proposals can be justified again by analogy from probability theory, based on the ratio (2).

As you know, in the classic production of the game, the top price of the game is determined as the best guaranteed result of the first player. In the fuzzy formulation of the guaranteed result cannot speak, but you can enter a different interpretation of the top price of the game. This is the result of the first player, if he will act in the best way, and the second player will act unsuccessfully, i.e. for some reason choose the worst strategy.

Definition 9. Let's denote the best result of the first player as $\max \widetilde{R}_{k}^{T r}$, and the worst result of the second $\max \widetilde{H}_{q}^{T r}$, then the fuzzy top price of the game

$$
\widetilde{\beta}=\max \widetilde{H}_{q}^{T r} \cap \max \widetilde{R}_{k}^{T r}, k=\overline{1, n}, q=\overline{1, m} \text {. }
$$

Definition 10. The fuzzy lower price of the game is determined based on their assump-tion that the second player, who is supposed to usually lose, chooses the best strategy $b_{l} \rightarrow \min _{l} \widetilde{H}_{q}^{T r}$, and the first - the worst strategy for him $a_{p} \rightarrow \min _{p} \widetilde{R}_{p}^{T r}$. Then

$$
\widetilde{\alpha}=\min \widetilde{H}_{l}^{T r} \cap \min \widetilde{R}_{p}^{T r} .
$$

It can be proved by using the comparison procedures described above that the ratio is true $\widetilde{\alpha} \leq \widetilde{\gamma} \leq \widetilde{\beta}$. 


\section{Numerical Example}

Consider a game with fuzzy payment matrices, the values of their elements were chosen arbitrarily from various sources, various options for membership functions are used, which were also chosen arbitrarily without any additional considerations.

Table 2. The fuzzy payment matrices

\begin{tabular}{|c|c|c|c|c|}
\hline & $b_{1}$ & $b_{2}$ & $b_{3}$ & $b_{4}$ \\
\hline$a_{1}$ & $\widetilde{10.6}($ peak $)$ & $\widetilde{15.6}($ trapeze $)$ & $\widetilde{15.6}($ triangle $)$ & $\widetilde{9.6}($ tent $)$ \\
\hline$a_{2}$ & $\widetilde{15.6}($ tent $)$ & $\widetilde{14.6}($ trapeze $)$ & $\widetilde{0.6}($ peak $)$ & $\widetilde{15.6}$ (trapeze) \\
\hline$a_{3}$ & $\widetilde{13.6}$ (trapeze) & $\widetilde{9.6}($ peak $)$ & $\widetilde{8.6}($ tent $)$ & $\widetilde{0.6}$ (trapeze) \\
\hline$a_{4}$ & $\widetilde{14.6}$ (triangle) & $\widetilde{9.6}$ (peak) & $\widetilde{10.6}$ (peak) & $\widetilde{13.6}$ (triangle) \\
\hline
\end{tabular}

In table $3 a_{1}, \ldots, a_{4}$ - strategies of the first player; $b_{1}, \ldots, b_{4}-$ strategies of the second player; elements of the payment matrix are fuzzy numbers, as indicated by the sign "wave" above the corresponding number, with symmetric membership functions, the type of which is indicated in brackets in the table cells; numeric values specified in the table cells under the sign "wave" are modal values of the corresponding fuzzy numbers.

For all calculations, a fuzzy table FuzzyCalc was used. A saddle point check showed its absence, a dominance check determined that the strategy $a_{3}$ is not useful and is excluded from consideration.

Figure 3 and Figure 4 show the membership functions of fuzzy estimates (numbers) of the consequences of the first player's choice of strategy $a_{1}$ (Fig. 3) and the second player - strategies $b_{3}$ (Fig. 4) and the results of applying the FztoTriangle transformation to these estimates. In both figures, combinations of elements corresponding to line $a_{1}$ (Fig. 3) and column $b_{3}$ (Fig. 4 ) of table 3 are shown in brackets.

Based on theorem 3, as a result of applying the FztoTriangle transformation to the lines (strategies of the first player) of table 3, equivalent fuzzy sets with triangular membership functions with parameters will be constructed:

for stratgy $a_{1}-z_{L}=9.3, z^{*}=14.66, z_{C G}=13.63, z_{R}=16.5$;

$a_{2}-z_{L}=0, z^{*}=16.5, z_{C G}=12.74, z_{R}=16.5$

$a_{3}-z_{L}=9.3, z^{*}=14.07, z_{C G}=13.06, z_{R}=16.5$.

Using the method of comparing fuzzy numbers proposed in (Chernov, 2018), we get that for the first player, the most preferred strategy is $a_{1}$, as a result of which the first player can expect the best result.

Similarly for the second player for strategies:

$b_{1}-z_{L}=10.2, z^{*}=14.53, z_{C G}=13.58, z_{R}=15.4$;

$b_{2}-z_{L}=9.3, z^{*}=16.5, z_{C G}=14.58, z_{R}=16.5$

$b_{3}-z_{L}=0, z^{*}=13.02, z_{C G}=10.06, z_{R}=16.5$

$b_{4}-z_{L}=9.3, z^{*}=15.63, z_{C G}=14.02, z_{R}=16.5$

accordingly, the best strategy of the second player will be $b_{3}$, as a result of which he can expect to lose the least.

For the specified payment matrix $\widetilde{\alpha}=\widetilde{10.85}<\widetilde{\gamma}=\widetilde{13.42}<\widetilde{\beta}=\widetilde{13.86}$. 


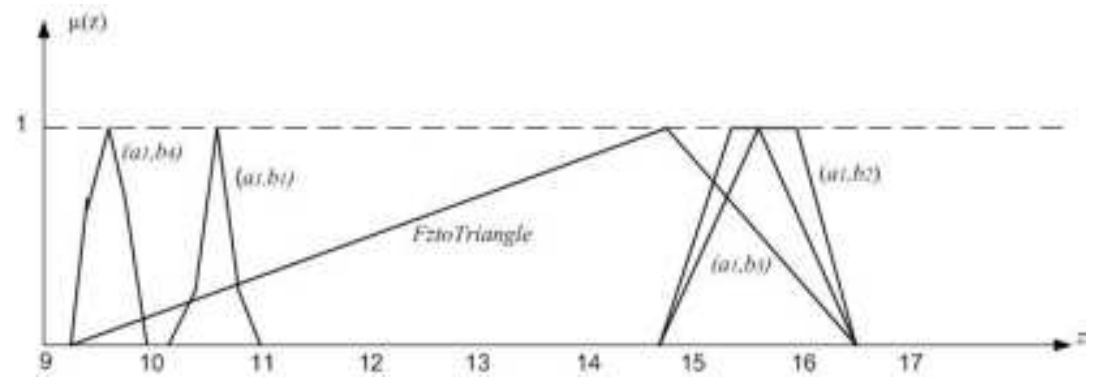

Fig. 3. Membership function for strategy $a_{1}$ first player after conversion FztoTriangle

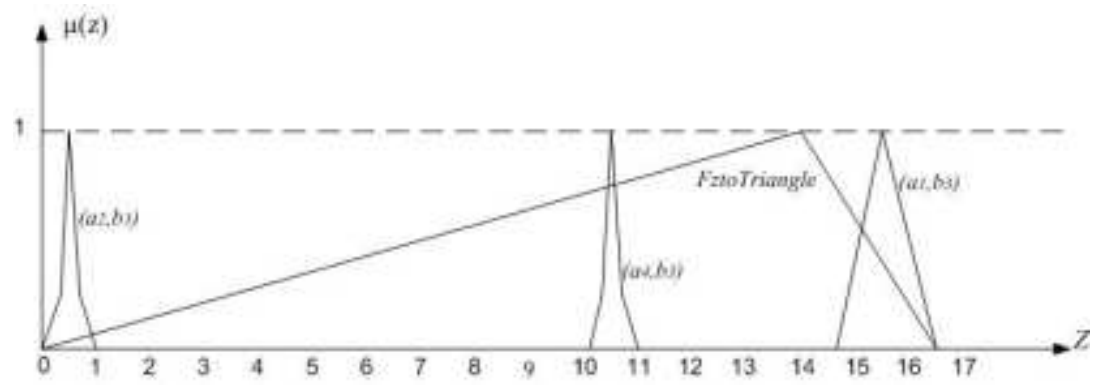

Fig. 4. Membership function for strategy $b_{3}$ second player after conversion FztoTriangle

\section{Conclusion}

The question arises, how to interpret the result. The value of the centroid or modal value obtained as a result of transformations should not be regarded as a result that will necessarily be obtained. It can be obtained from the set of results determined by the fuzzy payment matrix, when applying the best strategy and some actions of the enemy, if the latter is within the framework of useful strategies.

The proposed method of solving the game with FPM allows you to find the best strategies of players without going to a clear interpretation of the game.

\section{References}

Bector, C. R. and S. Chandra (2010). Fuzzy Mathematical Programming and Fuzzy Matrix Games. Springer.

Campos, L. (1989). Fuzzy linear programming model to solve fuzzy matrix games. Fuzzy Sets and Systems, 32(3), 275-289.

Cevikel, A.C. and M. Ahlatcloglu (2010). Solution for fuzzy matrix games. Computer and Mathematics with Applications, 60, 399-410.

Chaker, A., A. C. Dariani and C. Lucas (2008). How can fuzzy logic determine game equilibriums better. Intelligent Systems (IS'08), 4-th International IEEE Conference, 1, 2-51-2-56.

Chang, P.-T. (1994). Ranking of Fuzzy Sets Based on the Concept of Existence. Computers and mathematics with applications, 27, 1-21.

Chernov, V. G., I. A. Andreev, D. A. Gradusov and D. V. Tretyakov (1998). The solution of business problems by means of fuzzy algebra. Torah-Center: Moscow (in Russian). 
Chernov, V.G. (2018). Comparison of fuzzy numbers based on the construction of a linear relationship of order. Dynamics of complex systems - XXI century, 2, 81-87 (in Russian).

Dubois, D. and H. Prade (1980). Theorie des Possibilites. Applications a la representation des conisisancesen in for natique. Masson.

Dutta, B. and S. Gupta (2006). On Nash equilibrium strategy of two-person zero-sum games with trapezoidal fuzzy payoffs. Fuzzy Information and Engineering, 6(3), 299-314.

Falomkina, O. V. (2009). Statistical fuzzy games. MGU: Moscow. (in Russian)

Higast, M. and G.J. Klir (1983). Measures of uncertainty and information based of possibility distribution. Int. J. General Systems, 9(1), 43-58.

Orlovsky, S. A. (1976). Games in a fuzzy environment. Journal of Computational Mathematics and Mathematical Physics, 6(16), 1427-1435 (in Russian).

Piegat, A. (2013). Fuzzy modeling and control. Physica: Verlog.

Qiu, D. Y. Xing and S. Chen (2018). Solving fuzzy matrix games through a ranking value function method. J. Math. Computer Sci., 18, 175-183.

Rao, P.P.B. and N.R. Shankar (2012). Ranking generalized fuzzy numbers using area, mode, spread and weight. International Journal of Applied Science and Engineering, $\mathbf{1 0}(\mathbf{1}), 41-57$.

Sahoo, L. (2015). Effect of defuzzification methods in solving fuzzy matrix games. Journal of New Theory, 8, 51-64.

Sahoo, L. (2017). An approach for solving fuzzy matrix games using signed distance method. UK Journal of Information and Computing Science, 12(1), 73-80.

Seagal, A. V. (2011). Game-theoretic model of investment decision making. Scientific notes of the Taurida National University named after V. I. Vernadsky, a series of "Economics and Management", 1(63), 193-205.

Seikh, M.R., P.K. Nayak, and M. Pal (2015). An alternative approach for solving fuzzy matrix games. International Journal of Mathematics and Soft Computing, 5(1), 79-92.

Seraya, O. V. and T.N. Katkova (2012). The task of the theory of games with a fuzzy payment matrix. Mathematical Machines and Systems, 3, 29-36.

Stalin, T. and M. Thirucheran (2015). Solving Fuzzy Matrix Games Defuzzificated by Trapezoidal Parabolic Fuzzy Numbers. International Journal for Scientific Research and Development, 3(10), 1006-1010.

Vasilevich, L.F. (2010). Solution of fuzzy matrix games. zavantag.com /docs/2010/index21314.html

Ventzel, E.S. (2004). Operations research. Tasks, principles, methodology. Drofa: Moskow (in Russian).

Vovk, S.P. (2012). A game of two persons with fuzzy strategies and preferences. Almanac of modern science and education, 7(85), 479-485 (in Russian).

Yager, R.R. (1977). Multi-objective decision-making using a fuzzy sets. Intern. J. ManMachine Studies, 9(4), 375-382.

Zaichenko, Yu.P. (2010). Game models of decision making in conditions of uncertainty. In: Proceedings of the $\mathrm{V}$ international seminar school "Theory of decision-making". Uzhgorod (in Ukrainian). 